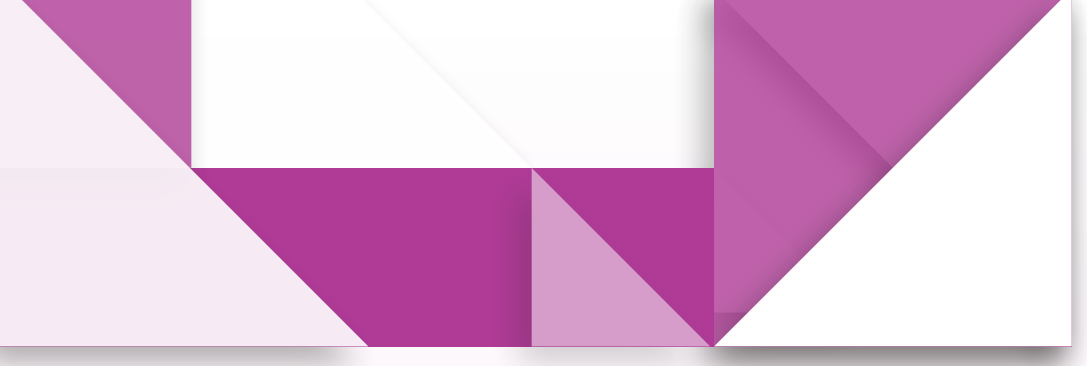

\title{
1) Efectos de una experiencia de campo sobre el gusto por la biología*
}

\section{- Effects of a Field Experience on the Love for Biology \\ - Efeitos de uma experiência de campo sobre o aprendizagem em biologia}

\section{Resumen}

Este estudio explora el efecto de una experiencia de aprendizaje sobre el gusto y las preferencias personales hacia la biología de un grupo de 32 niños de cuarto grado de primaria de un colegio de una ciudad de aproximadamente 200 mil habitantes de Colombia en edades entre los 9 y 10 años. Los resultados hacen parte de un estudio sobre el aprendizaje de la taxonomía y de la biología en ambientes informales de aprendizaje. Este reporte de caso educativo tiene como objetivo revisar los efectos que tiene la visita al ambiente informal de aprendizaje del herbario de la Universidad Pedagógica y Tecnológica de Colombia sobre preferencias y gustos básicos sobre este conocimiento. La experiencia de aprendizaje sigue los lineamientos de los experimentos de diseño (design experiments) en los cuales se elaboró una situación educativa y se evaluaron los efectos de manera cualitativa, utilizando una codificación estructurada. Para el estudio se comparan las respuestas de los estudiantes antes y después de la intervención con una entrevista semiestructurada. La intervención mostró que el contacto con el ambiente del herbario y con las prácticas de la biología favorece el gusto de los niños hacia el dominio disciplinar de la biología y genera un mayor entendimiento del quehacer del biólogo.

Palabras clave

educación informal; identidad; educación ambiental; biología; cognición
Lady Mayerli Vergara Estupiñán** Javier Alejandro Corredor Aristizábal ${ }^{* * *}$

** Magíster en Psicología, profesor de la Universidad Pedagógica y Tecnológica de Colombia, Escuela de Psicología, Tunja, Colombia.

lady.vergara@uptc.edu.co

** Doctor en Cognitive Studies in Education, profesor asociado en dedicación exclusiva en la Universidad Nacional de Colombia, Departamento de Psicología, Bogotá, Colombia.

jacorredora@unal.edu.co
Los hallazgos aquí reportados hacen parte de la tesis de maestría Aprendiendo sobre taxonomía y biología en ambientes informales de aprendizajes desarrollada con la Universidad Nacional de Colombia.

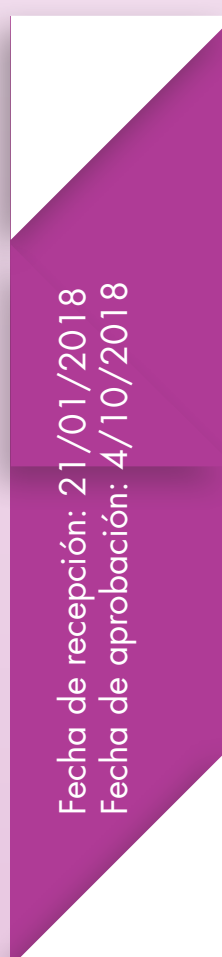




\section{Abstract}

This study explores the effect of a learning experience on the love and personal preference for biology in a group of 32 fourth-grade children from a school in a Colombian city of approximately 200 thousand people between the ages of 9 and 10. The results are part of a study on the learning of taxonomy and biology in informal learning environments. This educational case report aims to review the effects of visiting the informal learning environment of the herbarium of Universidad Pedagógica y Tecnológica de Colombia on the preferences and liking for this knowledge. The learning experience follows the guidelines of the design experiments in which an educational situation was developed and the effects were evaluated qualitatively by using a structured coding. The students' answers before and after the intervention are compared with a semi-structured interview. The intervention showed that contact with the herbarium's environment and with the practices of biology favors the children's liking for the disciplinary knowledge of biology and generates a greater understanding of the biologist's work.

Keywords

Informal environment; personal preferences; expertise; islands of expertise

\section{Resumo}

Este estudo explora o efeito de uma experiência de aprendizagem sobre o amor e as preferências pessoais sobre a biologia de um grupo de 32 crianças da quarta série, em uma escola localizada em uma cidade de aproximadamente 200 mil habitantes da Colômbia, de entre 9 e 10 anos de idade. Os resultados são parte de um estudo sobre a aprendizagem de taxonomia e biologia em ambientes informais de aprendizagem. Este relatório de caso educacional tem como objetivo revisar os efeitos da visita ao ambiente informal de aprendizagem do herbário da Universidade Pedagógica e Tecnológica da Colômbia sobre as preferências dos alunos com respeito a esse conhecimento. A experiência de aprendizagem segue as diretrizes dos experimentos de design (design experiments) nos que estabelecemos uma situação educacional e avaliamos seus efeitos qualitativamente usando uma codificação estruturada. Para o estudo foram comparadas as respostas dos alunos antes e depois da intervenção com uma entrevista semiestruturada. A intervenção evidenciou que o contato com o ambiente do herbário e com as práticas de biologia favorece o interesse das crianças pelo domínio da biologia e gera uma maior compreensão do trabalho do biólogo.

Palavras-chave

ambiente informal; preferências pessoais; especialização; ilhas de experiência 


\section{Introducción}

El presente estudio busca determinar el efecto de una experiencia de aprendizaje en un ambiente informal sobre la motivación de un grupo de estudiantes de cuarto grado dentro del dominio disciplinar de la biología. La investigación se desarrolló en un colegio de una ciudad intermedia de Colombia con un grupo de 32 niños en edades entre los 9 y 10 años. Dentro de los alcances de la investigación se buscó establecer los efectos de esta experiencia de aprendizaje sobre el gusto y preferencias personales hacia la biología en un grupo de niños y niñas. Particularmente, el estudio evalúa estos efectos en el contexto de una visita al Herbario de la Universidad Pedagógica y Tecnológica de Colombia.

Esta investigación sigue los lineamientos de los experimentos de diseño (design experiments) en los cuales se elabora una situación educativa y se evalúan sus efectos de manera cualitativa (Cobb, Disessa, Lehrer y Schauble, 2003). Específicamente, este estudio compara las respuestas de los estudiantes a una serie de tareas antes y después de la intervención. Para la recolección de los datos se realiza una encuesta sobre los efectos de la intervención sobre el gusto por la biología. La encuesta (pretest) se aplica antes de la visita al ambiente informal del herbario, y se vuelve a aplicar al final de la intervención (postest). Este procedimiento permite identificar cambios en las preferencias personales alrededor del dominio de la biología.

En particular, la experiencia evalúa si los niños y niñas desarrollan un mayor entendimiento del papel de los biólogos y un mayor gusto hacia las prácticas de la biología a partir de una experiencia informal diseñada para tal fin. Dado que el proceso de aprendizaje que se estudia acá se produce en la interacción los niños y niñas con materiales teóricos (e. g. claves taxonómicas o libros de texto) y con el ambiente del herbario, se espera que los estudiantes cambien la manera en que conciben el gusto y la motivación hacia la biología. Esto es así porque las experiencias de aprendizaje no solo modifican los elementos cognitivos, sino también las ideas que tienen los estudiantes sobre la práctica y estructura de las disciplinas científicas (Greeno, Collins y Resnick, 1986).

En relación con el gusto hacia la biología, Eberbach y Crowley (2008) sugieren que la observación del mundo natural está determinada no solo por procesos puramente cognitivos sino también por los intereses de los individuos que participan en ella. Desde esta perspectiva, los biólogos tienen una identidad positiva hacia la observación biológica y un gusto especial por la organización del mundo natural, que constituyen disposiciones productivas para el desarrollo de la experticia en esta área (Eberbach y Crowley, 2008). En este sentido, el gusto se entiende, en este estudio, como una preferencia básica hacia un área de conocimiento que se asocia a la identidad propia de los niños y niñas (Boaler, 2002). Aunque este estudio tiene una extensión temporal demasiado corta para permitir explorar cambios profundos en la identidad, sí permite ver cambios en las preferencias personales, el gusto, hacia un campo determinado de saber y detectar cambios en el conocimiento de los estudiantes en relación con lo que la práctica académica y profesional de una disciplina implica.

\section{Marco teórico}

\section{Islas de experticia}

Un concepto clave en la comprensión de lo que sucede durante una experiencia informal de aprendizaje es el concepto de islas de experticia. Este concepto plantea que las habilidades de resolución de problemas no dependen de habilidades generales, sino del conocimiento acumulado en un dominio determinado. 
Particularmente, se sabe que los niños y niñas pueden desarrollar islas de experticia en áreas específicas a partir del fortalecimiento de los intereses de los niños y niñas en prácticas cotidianas. De esta forma, se asume que los padres o profesores actúan como mediadores entre los intereses del niño y lo que aprende. Al respecto, autores como Crowley y Galco (2001) introducen el concepto de explanatoid, que hace referencia a las explicaciones que ofrecen los padres a las preguntas que los niños y niñas realizan en actividades cotidianas. Los contenidos de estas preguntas pueden ser muy diversos y hacer referencia a acontecimientos naturales, organismos biológicos, génesis de los objetos, fenómenos físicos y procesos culturales, entre otros. Como señalan Callanan y Oakes (1992), en las conversaciones entre padres e hijos, los niños y niñas centran su interés en descubrir ¿̇por qué suceden las cosas? y żcómo funcionan las cosas? Crowley y Jacobs (2002) señalan la importancia que tienen los padres en la formación de "islas de experticia" en la construcción de explicaciones al resolver problemas y generar cambios conceptuales. Los autores indican que las explicaciones de los padres contribuyen a formar lo que los niños y niñas están aprendiendo en la medida en que satisfacen su curiosidad e incentivan aprendizajes nuevos.

El tipo de explicaciones (explanatoids) que ofrecen los padres está particularmente diferenciado de aquellas que se dan convencional o formalmente en otro tipo de situaciones (e. g. explicaciones científicas e instruccionales). Debido principalmente a que las explicaciones que dan los padres se caracterizan por estar presentes en un momento de colaboración entre ambos, ofrecer evidencia notable cuando se persigue un interés común, facilitar que los niños y niñas analicen, almacenen y establezcan inferencias y, en último lugar, permitir que ellos y ellas puedan coconstruir su pensamiento científico en ajuste a las actividades cotidianas (Crowley y Jacobs, 2002). Generalmente, se sabe que en ambientes informales o de museo, los padres median entre las experiencias dentro y fuera de estos y están continuamente ayudando a relacionar el conocimiento informal con las diferentes disciplinas académicas, partiendo de los intereses y de las experiencias particulares que sus hijos han tenido (Crowley y Jacobs, 2002; Leinhardt y Crowley, 2002).

Esto implica que los niños y niñas tengan algún tipo de conocimiento específico sobre un tema de interés para la familia. Una muestra de este tipo de estudios fue la desarrollada por los autores en el museo de los niños de Pittsburgh. A través de las conversaciones con padres, se mostró que el contenido de las conversaciones se centraba en ejemplos de situaciones pasadas donde los niños y niñas habían adquirido dicho conocimiento. Los ejemplos usados por los padres establecían una conexión entre el objeto que era expuesto en la exhibición y el conocimiento anterior adquirido en la experiencia previa de cada niño (Crowley y Jacobs, 2002).

En dicha investigación entre los padres y los hijos se llegaron a plantear hipótesis sobre qué era cada fósil y se consiguió identificar que a niveles altos de conversación los niños y niñas identifican más fósiles. Para los autores, un nivel 
alto de conversación se producía cuando esta giraba efectivamente alrededor de explicaciones y no alrededor de categorías de habla superficiales (niveles bajos de conversación). En esta medida, es importante señalar que en museos o ambientes informales se pueden unir los conocimientos que tienen los niños y niñas con objetos auténticos, lo que posibilita que el aprendizaje se construya de manera significativa y permita un mejor recuerdo de lo que se aprende (Crowley y Jacobs, 2002; Leinhardt y Crowley, 2002).

De forma similar, Leinhardt y Crowley (2002) señalan que las experiencias de aprendizaje informal, como por ejemplo las visitas a los museos, posibilitan que los aprendizajes sean más significativos debido principalmente a que las colecciones de objetos gozan de autenticidad y se convierten en el mejor apoyo para generar nuevos aprendizajes. Además, los objetos que se encuentran en el museo sirven para ser utilizados como ejemplo y se convierten en reforzadores del aprendizaje.

Los autores establecen que la asociación que se logra con los museos parte de las experiencias pasadas del individuo y del objeto mismo que es observado. Ellos destacan también la importancia que los padres y los profesores tienen en el uso activo de los objetos en las prácticas de aprendizaje, principalmente porque ellos se convierten en un punto de anclaje entre el conocimiento en ambientes informales y los objetos. Este tipo de conexión se logra gracias a las conversaciones que se propician alcanzándose así cohesión social y participación entre los espectadores. De esta forma, las conversaciones se convierten en mecanismos de aprendizaje.

En ambientes informales relacionados con el mundo biológico existe una clara posibilidad de que los niños y niñas desarrollen ciertos conocimientos sobre dominios específicos (islas de experticia) gracias a la observación, la comparación, la manipulación y la interacción con el medio en su cotidianidad. Eberbach y Crowley (2008) denotan que las observaciones científicas que un experto realiza pueden ser traslapadas a las observaciones que realizan los niños y niñas en su cotidianidad y que les permiten aprender sobre su mundo biológico.

En particular, Eberbach y Crowley (2008) sugieren que los niños y niñas al igual que los expertos desarrollan características propias de la observación científica lo que les permite adquirir un conocimiento específico en biología. Los niños y niñas, de manera similar a los expertos, realizan observaciones científicas, toman notas de sus observaciones, crean archivos sobre las observaciones realizadas y formulan disposiciones para observar.

\section{Aprendizaje colaborativo}

Está investigación utiliza los conceptos expuestos por Vygotsky (1978) en relación con el aprendizaje colaborativo, ya que el estudio explora la forma como los niños y niñas se comportan en un ambiente informal del herbario, y relaciona los cambios cognitivos que se dan en la interacción con los pares y con los expertos (biólogos). Para el estudio teórico se parte de la importancia que tiene el desarrollo social en la construcción de conocimiento. Se ha demostrado que la comunicación entre pares, con docentes o adultos ha permitido a los niños y niñas adquirir habilidades y conocimientos gracias a la interacción social. Según Piaget (1985), los conocimientos aumentan luego de la interacción entre pares, debido a la falta de control que se puede dar cuando el juego es dirigido por un adulto. Así mismo, autores como Bell, Grossen y Perret-Clermont (1985) concluyeron que en las tareas de conservación formuladas por Piaget, los niños y niñas obtenían mejores resultados cuando 
trabajan en grupo que cuando lo hacían solos. Sullivan (1998) sugiere que la clave de estas interacciones se da gracias a la comunicación verbal que tiene lugar en las actividades y en donde se presenta un claro cambio en las cogniciones de los participantes.

Al respecto, Vygotsky (1978) señala que el cambio en las cogniciones y en las estructuras se da en la interacción con los demás, lo que permite que en las actividades diarias el niño esté construyendo diferentes aprendizajes. Con la introducción de la teoría de zona de desarrollo próximo (ZDP), Vygotsky establece que el desarrollo intelectual de un niño depende de lo que este es capaz de hacer por sí mismo (nivel de desarrollo efectivo) y lo que es capaz de hacer con ayuda de un adulto o tutor (nivel de desarrollo potencial), así se garantiza la adquisición de nuevos aprendizajes.

Al respecto señala Sullivan (1998), retomando los postulados de Vygotsky, refiere que existen cuatro aspectos esenciales en el desarrollo del aprendizaje. El primero es el desarrollo filogenético que hace referencia al uso de herramientas por parte de los humanos y la adquisición de signos y símbolos en el lenguaje. El segundo nivel es el cultural que relaciona las prácticas exclusivas de cada grupo y su reproducción (Melo, 2017). El tercero es el nivel ontogénico que parte de las características individuales de cada persona, su temperamento, aptitudes y actitudes. Finalmente, el cuarto nivel es el microgenético que hace referencia a la interacción entre el individuo y su entorno y tiene en cuenta las relaciones interpersonales. En este sentido, las prácticas informales que se observan en este estudio modifican el nivel cultural al que se ve expuesto el individuo en desarrollo, afectando de esta manera los demás niveles de desarrollo.

\section{Identidad y experiencias informales}

Esta investigación explora el efecto que tiene una intervención educativa en ambientes informales sobre el gusto y la preferencia por la biología. Dado que la investigación es un experimento de diseño (design experiment) en el que se construye y evalúa una experiencia de aprendizaje en ambientes informales, se espera que los participantes cambien no solo en aspectos cognitivo, sino en aspectos relacionados con la motivación y el gusto hacia un área de desempeño, en este caso la biología. La literatura señala que el aprendizaje en contextos escolares trasciende el cambio conceptual y el desarrollo cognitivo (Greeno et al., 1986). Dentro y fuera de la escuela, los niños y niñas desarrollan identidades y roles asociados a las disciplinas y prácticas escolares (Boaler, 2002). Por ejemplo, la investigación previa muestra que niños y niñas expuestos a prácticas escolares tradicionales desarrollan identidades como consumidores y no como productores de conocimiento (Boaler, 1998). La investigación también señala que la exposición recurrente a interacciones con pares en situaciones que implican la resolución de problemas dentro de contextos disciplinares contribuye a la modificación de las identidades y epistemologías que sostienen los niños. Cuando los niños 
y niñas se exponen a estas situaciones, ellos entienden que pueden tener un papel activo en la construcción de conocimiento y asumen una disposición epistemológica en la cual el conocimiento es construido y no absoluto.

La intervención educativa que se utiliza en esta investigación tiene una dimensión temporal muy corta. En este sentido, es difícil proponer que la intervención va a producir cambios profundos en las identidades de los niños y niñas. Sin embargo, la experiencia informal de este estudio puede contribuir a que los niños y niñas adquieran elementos a partir de los cuales pueden desarrollar una identidad disciplinar. Experiencias informales como las visitas a museos, generan conexiones personales con los objetos y contenidos presentes en estos (Leinhardt, Tittle y Knutson, 2002). Por ejemplo, algunos visitantes se identifican con personajes históricos cuyas vidas son descritas en museos. A lo largo de visitas repetidas a museos, estos elementos transforman la experiencia de los visitantes y modifican su relación con los contenidos en formas que pueden ser consideradas transiciones identitarias, ya que hacen que los visitantes empiecen a considerar ciertos contenidos como fundamentales para ellos como personas (Leinhardt, y Gregg, 2002).

Adicionalmente, la literatura muestra que el surgimiento de identidades depende de las opciones disponibles en el ambiente a las cuales se desarrollan los niños y niñas (Coté y Levine, 2002). Por ejemplo, la baja presencia de modelos de rol en profesiones científicas para niños y niñas pertenecientes a minorías expli$c a$, en alguna medida, por qué los niños y niñas de estos grupos no toman decisiones profesionales y de proyecto de vida, asociadas a dichas áreas (Lockwood y Kunda, 1997). En el mismo sentido, la literatura reciente en enseñanza de la ciencia resalta la importancia de exponer a los niños y niñas a actividades que se asemejen a actividades de investigación científica auténtica, ya que estas permiten que las personas construyan una imagen realista y positiva de lo que implica la actividad científica (Schwartz, Lederman y Crawford, 2004). Este estudio, entonces, explora cómo el contacto con una experiencia informal relacionada con la biología permite el desarrollo de preferencias y gustos hacia dicha disciplina, así como un mayor conocimiento de las actividades que se realizan en ella.

\section{Metodología}

\section{Participantes}

El tipo de muestreo para seleccionar la muestra fue un criterio de conveniencia, determinado por la facilidad de acceso proporcionado por la institución educativa elegida. La muestra fue conformada por 32 niños y niñas que se encuentran cursando cuarto grado de primaria de un colegio de una ciudad intermedia de Colombia. El tamaño de la muestra se seleccionó de forma intencional en función de la disponibilidad del colegio, el cual se ubica en un área urbana. Los participantes tenían edades entre los 9 y 10 años de edad y pertenecen a los estratos socioeconómicos 1, 2 y 3. A los participantes se les informó del estudio, y a los padres se les envió un consentimiento informado donde se les daba a conocer el objetivo del estudio. En dicho consentimiento se les informó que se realizarían grabaciones de las ejecuciones de los estudiantes y que estas no serían presentadas públicamente. En la misma línea se incluyó información que garantizaba el retiro voluntario del proyecto y demás garantías éticas para la participación en el estudio. Adicionalmente, se les informó que se realizaría una salida al Herbario de la Universidad Pedagógica y Tecnológica de Colombia, y se les pidió que evaluando dichas condiciones dieran o no su consentimiento. 


\section{Instrumentos}

Entrevista. La entrevista que se diseñó para el estudio busca evaluar las preferencias personales en relación con el dominio de conocimiento en biología y el disfrute del contacto con el mundo natural.

La entrevista aplicada a los niños y niñas tiene preguntas de relación con el dominio disciplinar; estas interrogan al niño sobre las nociones que tiene de lo que es ser un biólogo y sobre las actividades que este realiza.

Experiencia de aprendizaje. La situación de aprendizaje está diseñada con dos actividades: la primera se desarrolla en los jardines del colegio donde los niños y niñas observan las plantas y sus partes y utilizan una guía de clasificación de hojas. La actividad es diseñada y realizada por el investigador y cuenta con la supervisión y asesoría de la docente de ciencias naturales del colegio. En esta actividad se instruye a los participantes sobre las partes de las plantas, sus funciones; esta se desarrolla en grupo de cinco estudiantes. Para las grabaciones realizadas de los diferentes momentos del estudio se da entrenamiento a un observador externo que no interviene en la investigación.

La segunda actividad se realiza en la visita guiada al Herbario de la Universidad Pedagógica y Tecnológica de Colombia. Las actividades de los niños y niñas se organizan alrededor de una guía de trabajo y de la interacción directa con los biólogos. Así los niños y niñas buscan grupos de plantas para incluir en una tarea de agrupamiento de hojas. Inicialmente, se pide a los niños y niñas que revisen de forma libre las plantas presentes en el herbario. Después se solicita a los niños y niñas que clasifiquen las hojas correspondientes de acuerdo con la guía de clasificación de hojas. Ya que la tarea es de tipo colaborativo, se les pide que realicen está actividad en grupos y discutan sus respuestas. Las discusiones son videograbadas y analizadas dentro del estudio. El resultado final de la tarea es una tabla donde se encuentra el nombre de la planta estudiada, el tipo de hoja y su dibujo.

En relación con el trasfondo pedagógico desde el cual se diseña la intervención utilizada en este experimento de diseño, el estudio parte del supuesto de que el desarrollo de los procesos cognitivos que subyacen la comprensión de la biología solo es posible en ambientes de aprendizaje que permiten la interacción colaborativa con pares y otros elementos del ambiente social.

\section{Procedimiento}

El estudio tuvo una duración cinco días. La recolección de datos para el estudio se desarrolla con la entrevista, que se aplicó al total de la muestra ( $n=32$ ) en un intervalo de dos días. Antes de la entrevista se inicia un diálogo entre el niño y el investigador, con el fin de aumentar la conexión personal y facilitar la entrada al grupo participante. 
En el tercer día de recolección de datos se realiza la intervención con la situación de aprendizaje y, finalmente (el quinto día), se aplica de nuevo la entrevista. Este estudio utiliza un enfoque descriptivo de métodos mixtos (cualitativo-cuantitativo), el cual combina la observación de campo con codificaciones numéricas. Se observa cómo una experiencia de aprendizaje que se desarrolla en el herbario tiene efecto sobre el gusto y las preferencias de un grupo de estudiantes de cuarto grado, en relación con el dominio disciplinar de la biología.

El propósito del método utilizado en este estudio es evaluar el cambio en sus preferencias personales alrededor del dominio de la biología. Se realiza una observación previa a la intervención y una nueva observación al final para determinar si se presenta algún tipo de cambio luego de la visita al ambiente informal de aprendizaje.

\section{Análisis de datos}

Atendiendo al enfoque descriptivo de métodos mixtos (cualitativo-cuantitativo) de la investigación, el análisis de los datos combina codificaciones numéricas con informes descriptivo-narrativos. Una vez recolectados los datos se inicia el análisis de las verbalizaciones de los niños y niñas en la entrevista. Las respuestas son transcritas a una matriz de Microsoft Excel y organizadas en tablas, para luego ser analizadas por el programa estadístico SPSS. El tratamiento estadístico de los datos se realiza a través de una prueba de $\mathrm{McNe}$ mar's para establecer la diferencia entre dos tratamientos correlacionados. Debido a que los procedimientos de la prueba McNemar's se distribuyen igual que la chi cuadrada, los estadísticos calculados se simbolizan como una chi cuadrada $\left(X^{2}\right)$. Para la presentación de los resultados se utilizan tablas de contingencia de 2 x 2 y de dos entradas de frecuencia, para cada niño y niña de la muestra y de los histogramas. En el caso del estudio se presentan los resultados de la entrevista aplicada antes y después de la experiencia de aprendizaje.

\section{Análisis y discusión de resultados}

\section{Cambios significativos en las concepciones y el gusto por la biología}

La intervención utilizada produjo cambios significativos en el entendimiento que los niños y niñas tienen de la profesión y el quehacer de los biólogos. Al comparar las respuestas dadas a las preguntas de la entrevista antes y después de la intervención (e. g. experiencia de aprendizaje), se logra establecer los efectos en el gusto de los niños y niñas hacia la biología. En las preguntas de relación con el dominio disciplinar se observa un mayor interés de los niños y niñas hacia la profesión del biólogo, aumento en el conocimiento sobre las tareas que realizan y gusto por la profesión.

Frente a la pregunta: ¿̇dónde trabajan los biólogos? se encontró que después la experiencia de aprendizaje se presentaron cambios significativos $\left(X^{2}=, 001 p<, 05\right)$ en las respuestas que dan los niños y niñas (véase tabla 1). Así, el $56 \%$ de los participantes con respuestas completas en la entrevista inicial aumentan al $87 \%$ luego de la intervención (véase figura 1). 


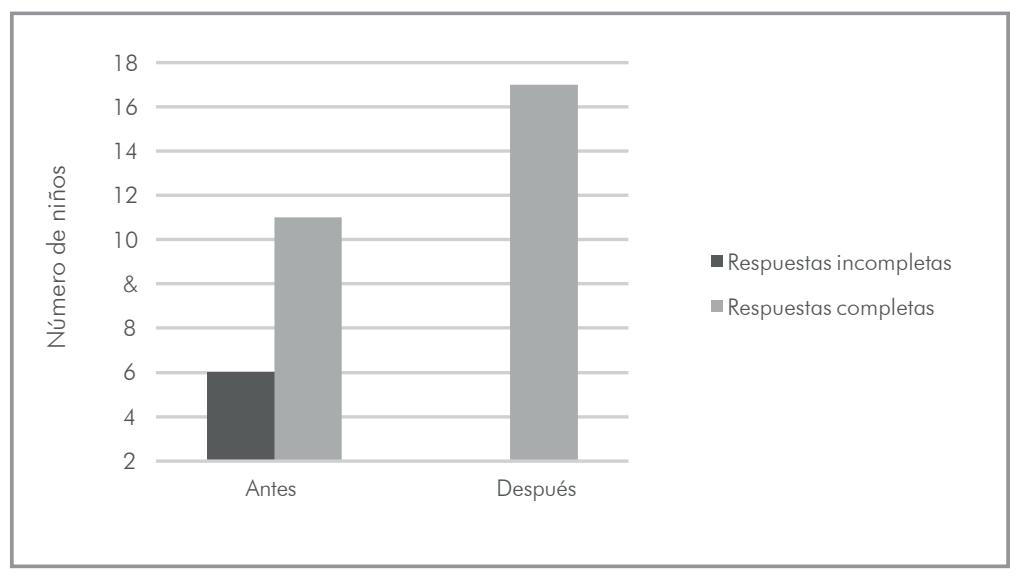

Figura 1. Respuestas a la pregunta: ¿̇dónde trabajan los biólogos?, antes y después de la experiencia de aprendizaje

Fuente: elaboración propia.

Luego de la visita al herbario las respuestas incluyen términos más cercanos al dominio disciplinar de la biología como herbario, laboratorio y trabajo de campo. Este tipo de términos advierten que el ambiente informal del herbario favorece la adquisición de un nuevo aprendizaje, da libertad para observar nuevos objetos y aclarar dudas. Además, los niños y niñas son capaces de establecer que en diferentes ambientes como el herbario, los laboratorios o en el campo, los biólogos se encargan de preservar las plantas.

Tabla 1. Contingencia de las respuestas antes y después de la experiencia de aprendizaje ¿̇Dónde trabajan los biólogos?

\begin{tabular}{|c|c|c|c|}
\hline \multicolumn{2}{|c|}{ No completas } & Completas & Total \\
\hline Antes & 4 & 11 & 15 \\
\hline Después & 0 & 17 & 17 \\
\hline Total & 4 & 28 & 32 \\
\hline
\end{tabular}

Fuente: elaboración propia.

De esta forma, es claro que con las experiencias de aprendizaje informal no solo modifican elementos cognitivos, sino también las ideas que los estudiantes tienen sobre la práctica y estructura de las disciplinas científicas para este caso sobre la biología (Greeno, Collins y Resnick, 1986). El que los participantes del estudio hayan tenido la posibilidad de interactuar en los jardines y en el herbario (i. e. con su mundo natural), garantiza mayor interés hacia la observación biológica y un gusto especial por la organización del mundo natural. Como señalan Eberbach y Crowley (2008), estas disposiciones especiales permiten el desarrollo de experticias en esta área de conocimiento, además de garantizar el inicio de procesos identitarios de aprendizaje más efectivos. 
En esta misma línea Cardona, Correa, Ríos y Sánchez (2017), con el estudio realizado en el Museo del Agua de Medellín, apoyan el uso de ambientes de aprendizaje no convencionales para favorecer el descubrimiento de la ciencia en los niños y niñas entre los 4 y 6 años, también, que este tipo de interacciones con objetos auténticos de aprendizaje garantizan que los niños y niñas sean sujetos activos en la construcción de la ciencia y se formulen diferentes hipótesis. Con este tipo de procesos se garantizan las habilidades necesarias para los dominios disciplinares propios de la biología.

Luego, al preguntarles a los niños y niñas sobre la idea de ser biólogo se encontró que hubo cambios significativos debido a la intervención ( $\left.X^{2}=, 008 p<, 05\right)$ (véase figura 2). Como se observa en la tabla 2, aumentó la disposición a ser un biólogo luego de la visita, lo que puede indicar que los aprendizajes vivenciales y ambientes colaborativos inciden en las preferencias de los niños y niñas. En otras palabras, se observó que los aprendizajes en ambientes colaborativos promueven el interés por las plantas gracias a la interacción con material auténtico, a su manipulación e interacción con los biólogos.

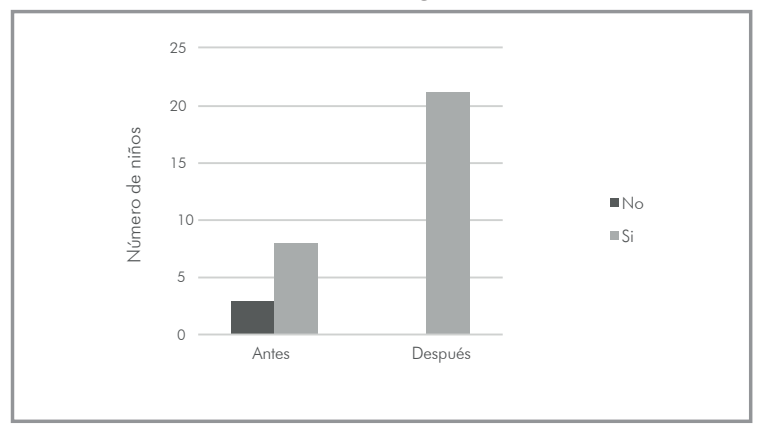

Figura 2. Respuestas a la pregunta: ¿̇te gustaría ser un biólogo? antes y después de la experiencia de aprendizaje

Fuente: elaboración propia.

En el caso particular de este estudio, este cambio respalda la idea de que experiencias pedagógicas alternativas tienen resultados en las prácticas pedagógicas tradicionales en el momento de motivar a los niños y niñas por el estudio de áreas de conocimiento científico. Es importante señalar que en ambientes informales o de museo las experiencias permiten relacionar el conocimiento informal con las diferentes disciplinas académicas, partiendo de los intereses y de las experiencias particulares que los niños y niñas han tenido (Crowley y Jacobs, 2002; Leinhardt y Crowley, 2002). De esta forma, el nivel de conversación gira alrededor de las explicaciones de los biólogos con los niños y niñas sobre la conservación de las plantas y permite que el aprendizaje se construya de manera significativa y garantiza un mejor recuerdo de lo que se aprende (Crowley y Jacobs, 2002; Leinhardt y Crowley, 2002).

Tabla 2. Contingencia de respuestas antes y después de la experiencia de aprendizaje $\dot{2} \mathrm{Te}$ gustaría ser un biólogo?

\begin{tabular}{|c|c|c|c|}
\hline \multicolumn{2}{|c}{ No } & \multicolumn{1}{c|}{ Sí } & Total \\
\hline Antes & 2 & 8 & 10 \\
\hline Después & 0 & 22 & 22 \\
\hline Total & 2 & 30 & 32 \\
\hline
\end{tabular}

Fuente: elaboración propia.

Dada la duración del estudio es muy difícil observar cambios en la identidad de los participantes, ya que estos requieren procesos sostenidos de cambio pedagógico y experiencia personal. Sin embargo, se observa la aparición de gustos y preferencias en relación con la biología. Estos gustos y preferencias no garantizan el surgimiento de identidades disciplinares positivas, pero sí son requisitos para que estas identidades surjan. Si bien la experiencia de aprendizaje tiene una extensión temporal corta son claros los cambios en las preferencias personales, el gusto y el conocimiento de los estudiantes en relación con la práctica profesional de una disciplina; así se garantiza la formación de islas de experticia sobre la biología (Crowley y Jacobs, 2002). 


\section{Salida al Jardín y visita al herbario}

En la salida al jardín del colegio, los niños y niñas tienen la posibilidad de identificar diferentes plantas con flores y utilizar una guía de la clasificación de hojas. Los niños y niñas muestran especial interés por observar, comparar, manipular e interactuar con las plantas del jardín, lo que permite que desarrollen ciertas habilidades relacionadas con el mundo biológico. Como señalan Eberbach y Crowley (2008), estas actividades propias de los expertos en biología pueden ser traslapadas a las observaciones que realizan los niños y niñas en su cotidianidad y que les permiten aprender sobre su mundo biológico. Estos autores sugieren que ellos al igual que los expertos desarrollan características propias de la observación científica lo que les permite adquirir un conocimiento específico en biología. La exploración del jardín les permitió a los niños y niñas — de forma similar a lo que los expertos realizan — tomar notas de sus observaciones y formular disposiciones para observar.

Una niña toca el centro de una flor blanca y les muestra el dedo a sus compañeras, dice "mira el polen". Ese mismo grupo de niñas refiere "esta es nuestra flor, la elegimos porque tiene mucho polen y es muy bonita, tiene tallo, raíz, tiene hojas, tiene pétalos, es gimnosperma, es una hoja entera... a ver, tiene pétalo... pero no tiene sépalo".

Los niños y niñas se fijan en los rasgos superficiales de las plantas, lo que no les permite aumentar sus niveles de experticia en relación con la tarea de identificación de la forma de las hojas. Al mismo tiempo, muestran interés y emoción por los aspectos de organización y funcionamiento las plantas en el jardín, lo que puede explicar el aumento en el gusto por el área de la biología.

En la visita al herbario los participantes tienen la posibilidad de interactuar con los biólogos y observar algunas plantas de la colección. Dentro la visita, los niños refieren que un herbario es "donde hay plantas y donde se exploran las plantas". Las explicaciones de los biólogos sobre qué es un herbario se basan en ejemplos que se conectan con sus experiencias cotidianas.

Un herbario es una colección de plantas. Allí se almacenan plantas. A lo largo de la carrera realizamos salidas de campo y observaciones de cómo son las plantas, si son de flores rojas, de flores amarillas. Luego traemos esas plantas al herbario, las secamos en el horno y hacemos todo un procedimiento para que ese material que traemos de campo quede en la colección del herbario.

Así, las explicaciones que dan los biólogos se caracterizan por estar presentes en un momento de colaboración, facilitar que los niños y niñas analicen la nueva información, ofrecer evidencia y permitir que los niños y niñas puedan coconstruir su pensamiento científico en ajuste a las actividades cotidianas (Crowley y Jacobs, 2002). Los biólogos se convierten en un punto de anclaje entre el conocimiento en ambientes informales y los objetos que se encuentran en el herbario. Este tipo de conexión se logra gracias a las conversaciones que se propician alcanzándose 
una cohesión social y participación entre los niños y niñas. De esta forma, las conversaciones se convierten en mecanismos de aprendizaje y los objetos del herbario en reforzadores de ese mismo aprendizaje. Además, las explicaciones de los biólogos sobre el proceso de conservación de las plantas se relaciona con el uso de explanatoid (Crowley y Galco, 2001), en la medida en que se dan explicaciones sobre el proceso de conservación de las plantas y se relacionan con las experiencias cotidianas de los niños y niñas.

La construcción de esas explicaciones permite resolver interrogantes y generar cambios conceptuales en los niños y niñas. Las explicaciones de los biólogos en la experiencia de aprendizaje contribuyen a formar lo que los niños y niñas están aprendiendo en la medida en que satisfacen su curiosidad e incentivan aprendizajes nuevos.

\section{Conclusiones}

La investigación logra establecer que hay ciertos efectos del contacto con el mundo natural que aumentan el gusto de los niños y niñas hacia la biología y su entendimiento. El aumento del interés de los niños y niñas por la naturaleza se evidencia en la tarea de exploración de plantas desarrollada en la experiencia de aprendizaje. Se propicia en los niños y niñas un mayor entendimiento del trabajo del biólogo e interés en la profesión. Con la experiencia del ambiente informal, los niños y niñas recrean tareas auténticas de investigación científica que realizan los biólogos; este tipo de actividades les permite formular hipótesis, exponer conceptos, preguntar a expertos, manipular plantas, realizar observaciones estructuradas, realizar clasificaciones y compartir con pares sus experiencias.

Aunque la intervención es corta refuerza en los niños y niñas conceptos adquiridos anteriormente y aumenta el gusto en el dominio disciplinar de la biología. Estos cambios son importantes porque señalan que la experiencia del herbario permitió que los niños y las niñas entendieran el papel profesional de los biólogos, que en cierta medida es un requisito para el desarrollo de identidades asociadas a los dominios disciplinares de las ciencias naturales. Lo que indica que en el ambiente informal del herbario se favorece la adquisición de nuevos aprendizajes, se les da libertad a los niños y niñas para observar nuevos objetos y aclarar dudas (Leinhardt y Crowley, 2002). Sullivan (1998) sugiere que este tipo de intereses surgen por la interacción entre pares y gracias a la comunicación verbal.

En la visita al herbario las relaciones e intercambios entre los niños y las niñas, y los biólogos, promueve diálogos, incentiva la construcción de procesos intelectivos a partir del otro, genera espacios de trabajo colaborativo y se posibilita la mediación en los procesos de enseñanza; de esta forma, los ambientes informales de aprendizaje son escenarios que generan el descubrimiento y la exploración, además de que permiten vivir experiencias significativas para los procesos de aprendizaje. Siguiendo la línea de los postulados expuestos por Vygotsky (1978), en relación con el aprendizaje colaborativo, el estudio permite ver como los niños y niñas interactúan con pares y con expertos (biólogos), se construyen conocimientos a partir de las interacciones sociales y se adquieren habilidades.

En el estudio, los niños y niñas asocian tareas que realizan los biólogos con laboratorios y salones donde se investiga con lupas y con experimentos; este tipo de tareas les interesan a los niños y niñas debido a que en el estudio se recrean tareas similares a las del biólogo. Como señalan Crowley y Jacobs (2002) y Leinhardt y Crowley (2002), en museos o ambientes informales se pueden unir los conocimientos que tienen los niños y niñas con 
objetos auténticos, lo que facilita que el aprendizaje sea significativo y permita un mejor recuerdo de lo que se aprende. Así, la tarea de recolección de la planta sirve de apoyo para mostrar cómo se coleccionan las plantas en el herbario y se convierte en un elemento auténtico de las prácticas que realiza el biólogo, lo que garantiza el desarrollo de dominios específicos de conocimiento. Esto, además, nos lleva a reflexionar sobre la potencia didáctica de las salidas educativas en las cuales los estudiantes no solo se exponen a nuevas experiencias, sino que el conjunto del contrato pedagógico se ve transformado al permitir, entre otras cosas, una exploración directa y autodirigida por parte de los estudiantes y otro tipo de relación menos jerárquica entre el docente y los estudiantes. En este sentido, se requiere que los docentes estén preparados para dirigir este tipo de actividades, lo cual implica por lo menos dos requisitos básicos: el primero es un conocimiento disciplinar adecuado que les permita guiar a los estudiantes a través de experiencias disciplinares auténticas; el segundo es la capacidad de entender que en este tipo de experiencias las relaciones tradicionales de la escuela necesitan ser modificadas de una trasmisión vertical de conocimiento hacia un acompañamiento en el proceso de descubrimiento.

Estos resultados son importantes porque muestran que experimentos de diseño (design experiments) que proporcionan contacto con el mundo natural pueden cambiar las preferencias y gustos de los niños y niñas, de manera que pueden actuar como requisito para el desarrollo de identidades cercanas a las disciplinas científicas. En contraste con las pedagogías tradicionales, la intervención expuesta en el estudio favorece el desarrollo de islas de experticia en biología en los niños y niñas, a partir de la exploración y observación del mundo natural favorecida por los padres y los profesores (Crowley y Jacobs, 2002). En este sentido, es importante recalcar cómo la transformación de las identidades y, más específicamente, de los gustos, las valoraciones y los comportamientos son un elemento fundamental en la transformación de la enseñanza de la biología. Esto es, la enseñanza de la biología debe trascender el espacio de la mera transmisión de contenido y enfocarse también en una transformación integral de las preferencias de los estudiantes, del sentido de sí mismo, siguiendo la propuesta de la perspectiva situada de que aprender es convertirse en otro (Larreamendy-Joerns, 2011).

Es evidente que en las tareas de jardinería de la familia se consolidan diferentes conocimientos sobre el cuidado de las plantas y su uso. Los padres, los profesores y, en el caso del estudio, los biólogos, permiten un uso activo de los objetos en las prácticas de aprendizaje, esto gracias a las interacciones con las colecciones de las plantas, los andamiajes y los diálogos que se generan en el ambiente informal y que consolidan nuevos conocimientos. La práctica de aprendizaje colaborativo en el herbario permite que profesores y biólogos ofrezcan explicaciones a los niños y niñas [explanatoids] para resolver los problemas de la tarea de clasificación (Crowley y Jacobs, 2002). Como señala Vygotsky (1978), el cambio en este tipo de cogniciones se da en las interacciones con los demás, en este caso, con la familia que permite, desde las actividades diarias, construir diferentes aprendizajes sobre el cuidado de las plantas con las tareas de jardinería. 
La experiencia de este estudio puede ampliar las opciones disponibles para los niños y niñas al exponerlos a experiencias asociadas con la biología y proveerlos de conocimiento sobre la profesión de los biólogos y la investigación en biología. Estos elementos, si bien no garantizan una identidad asociada a la disciplina, si aumentan el espectro de mundos posibles asociados a esta de los que los niños y niñas pueden escoger. En ese sentido, esta experiencia puede no mostrar cambios identitarios pero sí determinar mayor gusto por el dominio, y mayor conocimiento sobre lo que la biología como profesión implica, ambos requisitos para el desarrollo de identidades.

\section{Referencias}

Bell, N., Grossen, M. y Perret-Clermont A. N. (1985). Sociocognitive conflict and intellectual growth. See Berkowitz (pp. 41-54).

Boaler, J. (1998). Open and closed mathematics: Student experiences and understanding. Journal for Research in Mathematics Education, 29(1), 41-62.

Boaler, J. (2002). The development of disciplinary relationships: Knowledge, practice and identify in Mathema-tics Classrooms. En J. Boaler (ed.), For the learning of Mathematics (pp. 42-47). Alberta: FLM Publishing Association.

Callanan, M. y Oakes, L. (1992). Preschoolers' questions and parents' explanations: Causal thinking in everyday activity. Cognitive Development, 7, 213-233. doi: 10.1016/08852014(92)90012-G

Cardona, M., Correa, M., Ríos, L. y Sánchez, Y. (2017). Actitudes hacia la ciencia en el preescolar mediante la implementación de una secuencia didáctica en un museo. Tecné, Espiteme y Didaxis: TED, 42, 115-124.
Coté, J. y Levine, C. (2002). Identity formation, agency, and culture: A social psychological synthesis. Mahwah, NJ: Lawrence Erlbaum.

Crowley, K. y Galco, J. (2001). Everyday activity and the development of scientific thinking. En K. Crowley, C. D. Schunn y T. Okada (eds.), Designing for Science: Implications from professional, instructional, and everyday science. Mahwah, NJ: Lawrence Erlbaum.

Crowley, K. y Jacobs, M. (2002). Building islands of expertise. In perspectives on object-centered learning in museums. En S. Paris (ed.), Multiple perspectives on object-centered learning (pp. $301-324)$. Mahwah, NJ: Lawrence Erlbaum.

Cobb, P., Disessa, A., Lehrer, R. y Schauble, L. (2003). Design experiments in educational research. Educational Researcher, 32(1), 9-13. doi: 10.3102/0013189X032001009

Eberbach, C. y Crowley, K. (2008). From every day to scientific: How children learn to observe the biologist's world. Review of Educational Research, 79(1), 39-68. doi: 10.3102/0034654308325899

Greeno, J., Collins A. y Resnick, L. (1986). Cognition and Learning. En D. Berliner, R. Calfee (comps.), Handbook of educational psychology (cap. 2, pp 15-46). Nueva York: Simon y Schuster Mcmillan.

Larreamendy-Joerns, J. F. (2011). Aprendizaje como reconfiguración de agencia. Revista de Estudios Sociales, (40), 33-43.

Leinhardt, G. y Crowley, K. (2002). Objects of learning, objects of talk: Changing minds in museums. En S. Paris (ed.), Multiple perspectives on object-centered learning (pp. 301 -324). Mahwah, NJ: Lawrence Erlbaum. 
Leinhardt, G. y Gregg, M. (2002). Burning buses, burning crosses: Student teachers see civil rights. En G. Leinhardt, K. Crowley y K. Knutson (eds.), Learning conversations in museums (pp. 139-166). Mahwah, NJ: Lawrence Erlbaum.

Leinhardt, G., Tittle, C. y Knutson, K. (2002). Talking to oneself: Diaries of Museum visits. En G. Leinhardt, K. Crowley y K. Knutson (eds.), Learning conversations in museums (pp. 103-134). Mahwah, NJ: Lawrence Erlbaum.

Lockwood, P. y Kunda, Z. (1997). Superstars and me: Predicting the impact of role models on the self. Journal of Personality and Social Psychology, 73(1), 91-103. doi: 10.1037/0022-3514.73.1.91

Melo, N. (2017). Los puentes en la enseñanza de las ciencias: un compromiso para comprender las investigaciones sobre las relaciones entre conocimientos científicos escolares y conocimientos ecológicos tradicionales. Tecné, Episteme y Didaxis, 42, 43-61.

Piaget, J. (1985). The equilibration of cognitive structures: The central problem of intellectual development (pp. 36-64). (T. Brown y K. L. Thampy, trads.). Chicago: University of Chicago Press. (From French).

Schwartz, R., Lederman, N. y Crawford, B. (2004). Developing views of nature of science in an authentic context: An explicit approach to bridging the gap between nature of science and scientific inquiry. Science Teacher Education, 88(4), 610 645. doi: $10.1002 /$ sce. 10128

Sullivan, A. (1998). Social constructivist perspectives on teaching and learning. Annual Reviews Inc. 49, 345-375.

Vygotsky, L (1978). Mind in society: The development of higher psychological processes. M. Cole, V. John-Steiner, S. Scribner, E. Souberman (eds.). Cambridge, MA: Harvard University Press.

\section{Para citar el artículo}

Vergara, L. y Corredor, J. (2019). Experiencia de aprendizaje. Tecné, Episteme y Didaxis: TED, 45, 175-190. 